\title{
Antiretroviral therapy and congenital abnormalities in infants born to HIV-infected women in the UK and Ireland, 1990-2007
}

Claire L Townsend, Barbara A Willey, Mario Cortina-Borja, Catherine S Peckham, Pat A Tookey

MRC Centre of Epidemiology for Child Health, UCL Institute of Child Health, University College London, London, United Kingdom

Corresponding author: Claire L Townsend, MRC Centre of Epidemiology for Child Health, UCL Institute of Child Health, University College London, 30 Guilford Street, London, WC1N 1EH, United Kingdom.

Tel: +44 2079052336 Fax: +44 $2079052381 \quad$ Email: c.townsend@ich.ucl.ac.uk

Running head: ART and congenital abnormalities

Word count: 1832

Tables: 2

Disclaimer: The views expressed in this paper are those of the authors alone. 
Data presented previously at the Royal College of Paediatrics and Child Health 12th Spring Meeting and published as an abstract in Archives of Disease in Childhood (2008; 93 (suppl_1): A75-A84. Abstract ALL/THUR/24).

Sources of financial support: The National Study of HIV in Pregnancy and Childhood is currently funded by the Health Protection Agency (grant number GHP/003/013/003). Claire Townsend is funded by the UK Medical Research Council (MRC) (grant number G0501895). This work was undertaken at the Centre for Paediatric Epidemiology and Biostatistics which benefits from funding support from the MRC in its capacity as the MRC Centre of Epidemiology for Child Health. The UCL Institute of Child Health receives a proportion of funding from the Department of Health's National Institute for Health Research Biomedical Research Centres funding scheme. Any views expressed in this paper are those of the authors, and not necessarily those of the funders. 


\section{Abstract}

Objective To explore the rate of reported congenital abnormalities in infants exposed to antiretroviral therapy (ART) in utero.

Design Comprehensive national surveillance study in the United Kingdom and Ireland.

Methods Births to diagnosed HIV-infected women are reported to the National Study of HIV in Pregnancy and Childhood. Infants born between 1990 and 2007 were included.

Results The rate of reported major and minor congenital abnormality was $2.8 \%$ (232/8242) overall, and there was no significant difference by timing of ART exposure: $2.8 \%(14 / 498)$ in unexposed infants, $2.7 \%$ (147/5427) following second or third trimester exposure, and 3.1\% (53/1708) following first trimester exposure $(p=0.690)$. There was no difference in abnormality rates by class of ART exposure in the first trimester $(p=0.363)$, and no category of abnormality was significantly associated with timing of ART, although numbers in these groups were small. There was no increased risk of abnormalities in infants exposed to efavirenz $(p=0.672)$ or didanosine $(p=0.816)$ in the first trimester.

Conclusions These findings, based on a large, national, unselected population provide further reassurance that ART in utero does not pose a major risk of fetal anomaly.

Keywords: Congenital abnormalities; HIV; Antiretroviral Therapy; Pregnancy; United Kingdom. 


\section{Introduction}

Antiretroviral therapy (ART) has had a major impact on reducing the risk of mother-tochild transmission of HIV, ${ }^{1}$ and the majority of HIV-infected women in the UK and Ireland now receive ART at some time in pregnancy. ${ }^{2}$ Nevertheless, there are concerns about potential teratogeneticity, particularly since around a quarter of HIV-infected women conceived on treatment in recent years. ${ }^{1}$

Large-scale observational studies have so far detected no overall increase in congenital abnormality rates associated with first trimester ART exposure. ${ }^{3-5}$ However, an increased abnormality rate in 353 infants exposed to didanosine in the first trimester $(4.5 \%, 95 \%$ confidence interval $[\mathrm{CI}]: 2.6 \%-7.3 \%)$ compared with a population rate of $2.7 \%$, was recently detected through international prospective monitoring by the Antiretroviral Pregnancy Registry, ${ }^{6}$ and a significantly increased prevalence of hypospadias following first trimester zidovudine exposure (7/382), compared with later or no exposure (2/892), has been reported from the Women and Infants Transmission Study (WITS). ${ }^{4}$ There have also been case reports of neural tube defects in infants exposed to efavirenz in the first trimester. ${ }^{7 ; 8}$

HIV infection in pregnant women and their children has been monitored in the UK and Ireland since the late 1980s, through a unique, population-based surveillance scheme. ${ }^{2}$ We previously investigated the association between in utero ART exposure and congenital abnormalities in over 3100 infants born between 1990 and $2003 .^{9}$ In this updated analysis, including four additional years of surveillance data, we report on over 8500 children born between 1990 and 2007. 


\section{Methods}

Active surveillance of obstetric and paediatric HIV in the UK and Ireland is carried out through the National Study of HIV in Pregnancy and Childhood (NSHPC). Full methods are described elsewhere. ${ }^{1 ; 2}$ This analysis includes all infants (live born, stillborn, twins and triplets) born between 1990 and 2007 in the UK and Ireland to women diagnosed before delivery, and reported by June 2008. Information on maternal demographic characteristics, pregnancy outcome, delivery, perinatal details, type and timing of ART is routinely collected through the NSHPC. Congenital abnormalities are reported by both obstetric and paediatric respondents, mostly within the first few weeks of life. Most variables are obtained from both sources, except for timing of ART and maternal clinical status (AIDS or HIV-related symptoms in pregnancy), which are collected only from obstetric respondents.

Timing of ART exposure in pregnancy was classified as early if therapy was started before conception or up to 12 completed weeks of gestation, and late if started after 12 weeks. Class of antiretroviral regimen was categorised as nucleoside reverse transcriptase inhibitors (NRTI) only, or according to inclusion of non-nucleoside reverse transcriptase inhibitors (NNRTI), protease inhibitors (PI), or both NNRTIs and PIs. Four infants in the PI group were also exposed to fusion inhibitors in the first trimester (none had an abnormality reported).

Congenital abnormalities were classified using the World Health Organisation's International Classification of Diseases. ${ }^{10}$ For infants with multiple abnormalities reported $(n=19)$, only the main abnormality was included in the analysis. Detailed 
information on whether abnormalities were major or minor was not routinely collected; however, in order to assess the prevalence of major abnormalities, the following were classified as minor: polydactyly, malformed ear, abnormalities of the feet, minor mouth abnormalities, undescended testes, accessory nipple, spinal hairy patch, strawberry nevi, skin tag, and subclinical sub-ependymal cysts.

Data were managed in a Microsoft Access 2002 database and analysed using Stata 10.0 (Stata Corporation, College Station, TX). Congenital abnormality rates and 95\% CIs were calculated. Because of specific concerns, abnormality rates were also calculated for infants exposed to efavirenz or didanosine in early pregnancy, as was the rate of hypospadias in male infants. Differences in rates were assessed using $\chi^{2}$ and Fisher's exact tests. Logistic regression models were developed to adjust for potential confounders, including maternal ethnicity, age, injecting drug use (as the reported route of HIV acquisition) and clinical status. ${ }^{11}$

\section{Results}

\section{Maternal and pregnancy characteristics and ART}

Altogether 8576 infants were reported, including 92 stillbirths and 288 twins or triplets. Information was available from both paediatric and obstetric sources for $79 \%$ of infants, but the remainder were reported only through the obstetric $(10 \%)$ or paediatric $(11 \%)$ scheme. Information on congenital abnormality was available for $96.1 \%(8242 / 8576)$ of infants. Of these, most were born between 2000 and 2007 (Table 1). Three quarters were born to black African women, and median maternal age at delivery was 30 years 
(interquartile range [IQR]: 26.3-33.8 years). Median age at last report was 6 months (IQR: 3-15 months).

Information on timing of ART exposure was missing for 7.4\% (609/8242) of infants, mostly $(88 \%, 538 / 609)$ because reports were only obtained from paediatric respondents who were not asked to provide this information. Less than a quarter of infants (22.4\%) had early in utero exposure (Table 1), mostly to NNRTI-containing regimens (52.9\%).

\section{Congenital abnormalities}

Altogether 232 infants out of 8242 were reported to have at least one congenital abnormality $(2.8 \%, 95 \%$ CI: $2.5 \%-3.2 \%)$, a quarter of whom had only minor abnormalities (59/232). The abnormality rate excluding minor defects was $2.1 \%$ (95\% CI: $1.8 \%-2.4 \%)$. Infants with missing information on congenital abnormality $(n=334)$ were more likely than those with information to be preterm or of low birth weight $(25 \%$ vs. $15 \%)$ or to have mothers who were untreated in pregnancy (30\% vs. $7 \%)(p<0.001)$, but not more likely to have early ART exposure ( $15 \%$ vs. $22 \%, p=0.478)$.

Abnormality rates were lower in infants born to black African mothers than in those born to white mothers, and higher among infants whose mothers were symptomatic (Table 1). Infants with abnormalities were more likely to be delivered prematurely and to be of low birth weight, and girls had a lower reported rate than boys.

There was no significant difference in the unadjusted abnormality rate by ART exposure: $2.8 \%$ in unexposed infants, $2.7 \%$ in those with late exposure, and $3.1 \%$ in those with early exposure $(p=0.690)$ (Table 1$)$. After adjusting for potential confounders (maternal ethnicity, age at delivery, injecting drug use and clinical status) neither late 
(adjusted odds ratio $[\mathrm{AOR}]=0.96,95 \% \mathrm{CI}: 0.54-1.71, p=0.889$ ) nor early ART exposure $(\mathrm{AOR}=1.01,95 \% \mathrm{CI}: 0.54-1.88, p=0.972)$ was significantly associated with congenital abnormality $(n=7179)$. AORs were similar to the unadjusted ORs presented in Table 1, including for maternal ethnic group and clinical status.

Class of ART was reported for $99.4 \%$ (1697/1708) of infants exposed in the first trimester and was not significantly associated with congenital abnormality $(p=0.363)$ (Table 1). After adjusting for maternal ethnicity, age, injecting drug use and clinical status, infants exposed to PIs showed no significant difference in congenital abnormality rates compared with those exposed to NNRTIs (AOR=1.09, 95\% CI: 0.58-2.07 $p=0.789$ ), nor did those exposed to NRTIs only (AOR=1.94, 95\% CI: 0.84-4.50, $p=0.123$ ), or NNRTI- and PI-containing regimens (AOR=1.15, 95\% CI: 0.34-3.92, $p=0.823)(n=1679)$. Infants exposed only to NRTIs were more likely than those exposed to other drug classes to be reported in earlier years and therefore to be born to young, white, drug-using women. ${ }^{2}$

A total of 220 infants were exposed to efavirenz, 205 (93.2\%) in early pregnancy; of those exposed early, 2.4\% (5/205) had abnormalities reported (undescended testes [n=2], hip dislocation [ $n=2]$, hypertrophic pyloric stenosis). This did not differ significantly from the rate in infants with first trimester exposure to drugs other than efavirenz $(3.2 \%, 48 / 1503, p=0.672)$. There were 284 exposures to didanosine, 174 $(61.3 \%)$ in the first trimester; of those infants with early exposure, $3.4 \%(6 / 174)$ had abnormalities reported (Down's syndrome, heart defect [ $n=2]$, hydronephrosis, jejunal atresia, foot abnormality); this did not differ significantly from the rate for other first trimester ART exposures $(3.1 \%, 47 / 1534, p=0.816)$. There were no abnormalities 
reported in infants only exposed to efavirenz $(n=15)$ or didanosine $(n=110)$ later in pregnancy.

The most commonly reported abnormality types were musculoskeletal (40/232), limb (32/232), heart/circulatory (30/232), and genital organs (22/232; 12 hypospadias, 9 undescended testes, 1 ambiguous genitalia) (Table 2). No category of abnormality was significantly associated with timing of ART (Table 2). Of the 12 cases of hypospadias, all were in infants exposed to zidovudine-containing regimens $(0.18 \%, 12 / 6711$, versus $0 \%, 0 / 792$, exposed to zidovudine-sparing regimens, $p=0.262$ ). Timing of ART was reported for 11 of these infants: two had early exposure (2/1708, $0.12 \%$; or $2 / 856$ boys, $0.23 \%)$, and nine late $(9 / 5427,0.17 \%$; or $9 / 2693$ boys, $0.33 \%)$, with no statistically significant difference between the two groups $(p=1.00)$.

Terminated pregnancies (not included in overall analysis)

Twenty-one congenital abnormalities were reported in 549 terminated pregnancies (1990-2007): anencephaly ( $n=4)$, Down's syndrome $(n=5)$, other chromosomal anomaly $(n=3)$, exomphalos, enlarged cerebral ventricles, cleft lip/palate, hydronephrosis, bowel abnormality, heart defect, spina bifida, achondroplasia, and renal agenesis. These 21 terminations were carried out between 12 and 30 weeks gestation, and only five were in women who were on treatment (HAART) in early pregnancy. The overall abnormality rate including these 21 terminations was $3.1 \%$ (253/8263, 95\% CI: 2.7-3.5).

\section{Discussion}


An overall congenital abnormality rate of $2.8 \%$ (2.1\% excluding minor defects) was observed in this unselected population of around 8200 infants. This is consistent with national population estimates of 2-3\% for major abnormalities in England and 2.2\% for Europe as a whole (calculated from EUROCAT data tables, 1980-2006). ${ }^{11 ; 2}$

In common with a number of other large-scale observational studies, ${ }^{3-5}$ we did not detect any significant association between rate of reported congenital abnormalities and type or timing of ART in pregnancy; nor did we detect any significant increase in the overall abnormality rate following early exposure to efavirenz $(n=205)$ or didanosine $(n=174)$. The number of reported cases of hypospadias was small $(n=12)$, and rates were similar following early or late ART exposure. The overall excess of abnormalities in boys was mainly accounted for by genital abnormalities, all of which were in boys. The increased abnormality rates in symptomatic and white women were reported previously ${ }^{9}$, but reasons remain unclear.

While these results are reassuring, the overall rate is likely to be a minimum estimate. In particular, abnormalities not apparent at birth might have been under-reported, either because the birth was only notified through the obstetric scheme, or because diagnosis occurred after the last report. However, reporting of most major abnormalities apparent in the first few weeks of life is likely to be relatively complete. Although ascertainment of early terminations in HIV-infected women is incomplete ${ }^{2}$, terminations carried out after a congenital anomaly scan are likely to be well reported.

Infants missing information on congenital abnormality were more likely to be premature, a factor associated with congenital abnormalities; however, they were no more likely to have early ART exposure that those with information provided. Finally, although information on maternal ethnicity, age, injecting drug use and clinical status 
was available, data on other potential confounders such as maternal smoking and diet during pregnancy, concurrent infections, and non-HIV medication were not.

Although this analysis included over 8200 infants (1700 with early ART exposure), at least 350 exposures to any specific drug would be required to detect a 2 -fold increase in overall risk with $80 \%$ power, and even larger numbers would be required to detect an association with a particular type of abnormality. Nevertheless, these results provide further reassurance that exposure to ART in utero does not pose a major risk of fetal anomaly. 


\section{Acknowledgements}

National surveillance of obstetric and paediatric HIV is undertaken through the National Study of HIV in Pregnancy and Childhood (NSHPC) in collaboration with the Health Protection Agency Centre for Infections, and Health Protection Scotland. We gratefully acknowledge the contribution of the midwives, obstetricians, genito-urinary physicians, paediatricians, clinical nurse specialists and all other colleagues who report to the NSHPC through the British Paediatric Surveillance Unit of the Royal College of Paediatrics and Child Health, and the obstetric reporting scheme run under the auspices of the Royal College of Obstetricians and Gynaecologists. We thank Janet Masters who co-ordinates the study and manages the data, and Icina Shakes and Kate Francis for administrative support. We also thank Hermione Lyall for her helpful comments on this paper.

\section{Disclosure of interests}

We declare that we have no conflicts of interest.

\section{Author contributions}

Barbara Willey and Claire Townsend carried out the statistical analyses with support from Mario Cortina-Borja, and jointly drafted the paper. All authors contributed to the interpretation of the results, commented on all drafts of the paper, and approved the final version. Pat Tookey is the guarantor.

\section{Ethics Approval}

Ethics approval for the NSHPC was renewed following review by the London Multi-

Centre Research Ethics Committee in 2004 (ref. MREC/04/2/009). 


\section{Funding}

The National Study of HIV in Pregnancy and Childhood is currently funded by the Health Protection Agency (grant number GHP/003/013/003). Claire Townsend is funded by the UK Medical Research Council (MRC) (grant number G0501895). This work was undertaken at the Centre for Paediatric Epidemiology and Biostatistics which benefits from funding support from the MRC in its capacity as the MRC Centre of Epidemiology for Child Health. The UCL Institute of Child Health receives a proportion of funding from the Department of Health's National Institute for Health Research Biomedical Research Centres funding scheme. Any views expressed in this paper are those of the authors, and not necessarily those of the funders. 
Table 1. Risk factors for congenital abnormalities in 8242 infants; rates and unadjusted odds ratios

\begin{tabular}{|c|c|c|c|c|c|c|c|}
\hline & & & Cong & & Una & ljusted Odds & \\
\hline & To & & abnor & & & Ratios & \\
\hline & $n$ & $\%$ & $n$ & $\%$ & OR & $(95 \% \mathrm{CI})$ & $p$-value \\
\hline Time period $(n=824$ & & & & & & & \\
\hline$\leq 1999$ & 833 & 10.1 & 31 & 3.7 & 1.00 & & \\
\hline $2000-2007$ & 7409 & 89.9 & 201 & 2.7 & 0.72 & $(0.49-1.06)$ & 0.970 \\
\hline Maternal character & & & & & & & \\
\hline Ethnic origin $(n=8$ & & & & & & & \\
\hline White * & 1285 & 15.7 & 46 & 3.6 & 1.00 & & \\
\hline Black African ** & 6244 & 76.4 & 162 & 2.6 & 0.72 & $(0.51-1.00)$ & 0.051 \\
\hline Black Other & 326 & 4.0 & 13 & 4.0 & 1.12 & $(0.60-2.10)$ & 0.726 \\
\hline Other & 316 & 3.9 & 10 & 3.2 & 0.88 & $(0.44-1.76)$ & 0.719 \\
\hline
\end{tabular}

Age at delivery $(n=8184)$

$\begin{array}{llllllll}<25 \text { years } & 1471 & 18.0 & 40 & 2.7 & 1.00 & & \\ 25-34 \text { years } & 5154 & 63.0 & 147 & 2.9 & 1.05 & (0.74-1.50) & 0.786 \\ \geq 35 \text { years } & 1559 & 19.0 & 43 & 2.8 & 1.01 & (0.66-1.57) & 0.948\end{array}$

HIV exposure group $(n=8242)$

Other risk $* * *$

Injecting drug use

$7876 \quad 95$

3664

Clinical status $(\boldsymbol{n}=\mathbf{7 2 3 5})$

$\begin{array}{lll}6451 & 89.2 & 174\end{array}$

$2.7 \quad 1.00$

HIV-related symptoms/AIDS

$\begin{array}{lll}219 & 2.8 \quad 1.00\end{array}$

$\begin{array}{lllll}13 & 3.6 & 1.29 & (0.73-2.28) & 0.384\end{array}$

Infant characteristics

$\operatorname{Sex}(n=8202)$

Male

$\begin{array}{lllll}4123 & 50.3 & 137 & 3.3 & 1.00\end{array}$

Female

$4079 \quad 49$ 
Gestational age $(n=8056)$

$\begin{array}{llllllll}\geq 37 \text { weeks } & 6874 & 85.3 & 182 & 2.6 & 1.00 & & \\ & & & & & & & \\ <37 \text { weeks } & 1182 & 14.7 & 47 & 4.0 & 1.52 & (1.10-2.11) & 0.012\end{array}$

Birth weight $(n=7153)$

$\begin{array}{llllllll}\geq 2.5 \mathrm{~kg} & 6067 & 84.8 & 158 & 2.6 & 1.00 & & \\ <2.5 \mathrm{~kg} & 1086 & 15.2 & 45 & 4.1 & 1.62 & (1.15-2.27) & 0.005\end{array}$

Treatment characteristics

Timing of ART exposure $(n=7633)$

$\begin{array}{lrrrrrrr}\text { Not treated in pregnancy } & 498 & 6.5 & 14 & 2.8 & 1.00 & & \\ \text { Late (2nd/3rd trimester) } & 5427 & 71.1 & 147 & 2.7 & 0.96 & (0.55-1.68) & 0.893 \\ \text { Early (1st trimester) } & 1708 & 22.4 & 53 & 3.1 & 1.11 & (0.61-2.01) & 0.739\end{array}$

Treatment class in 1st trimester $(n=1697)$

$\begin{array}{lccccccc}\text { NRTI only } & 148 & 8.7 & 8 & 5.4 & 2.08 & (0.92-4.72) & 0.080 \\ \text { NNRTI } & 898 & 52.9 & 24 & 2.7 & 1.00 & & \\ \text { PI } & 553 & 32.6 & 17 & 3.1 & 1.16 & (0.61-2.17) & 0.654 \\ \text { NNRTI \& PI } & 98 & 5.8 & 3 & 3.1 & 1.15 & (0.34-3.89) & 0.822\end{array}$

$* 82.5 \%(998 / 1210)$ of white women were born in the UK or Ireland; ** 97.6\% (6012/6162) of black African women were born in sub-Saharan Africa; *** Other risk includes heterosexual, 'from area of high HIV prevalence' and vertical transmission. 
Table 2. Reported category of congenital abnormality by timing of ART exposure

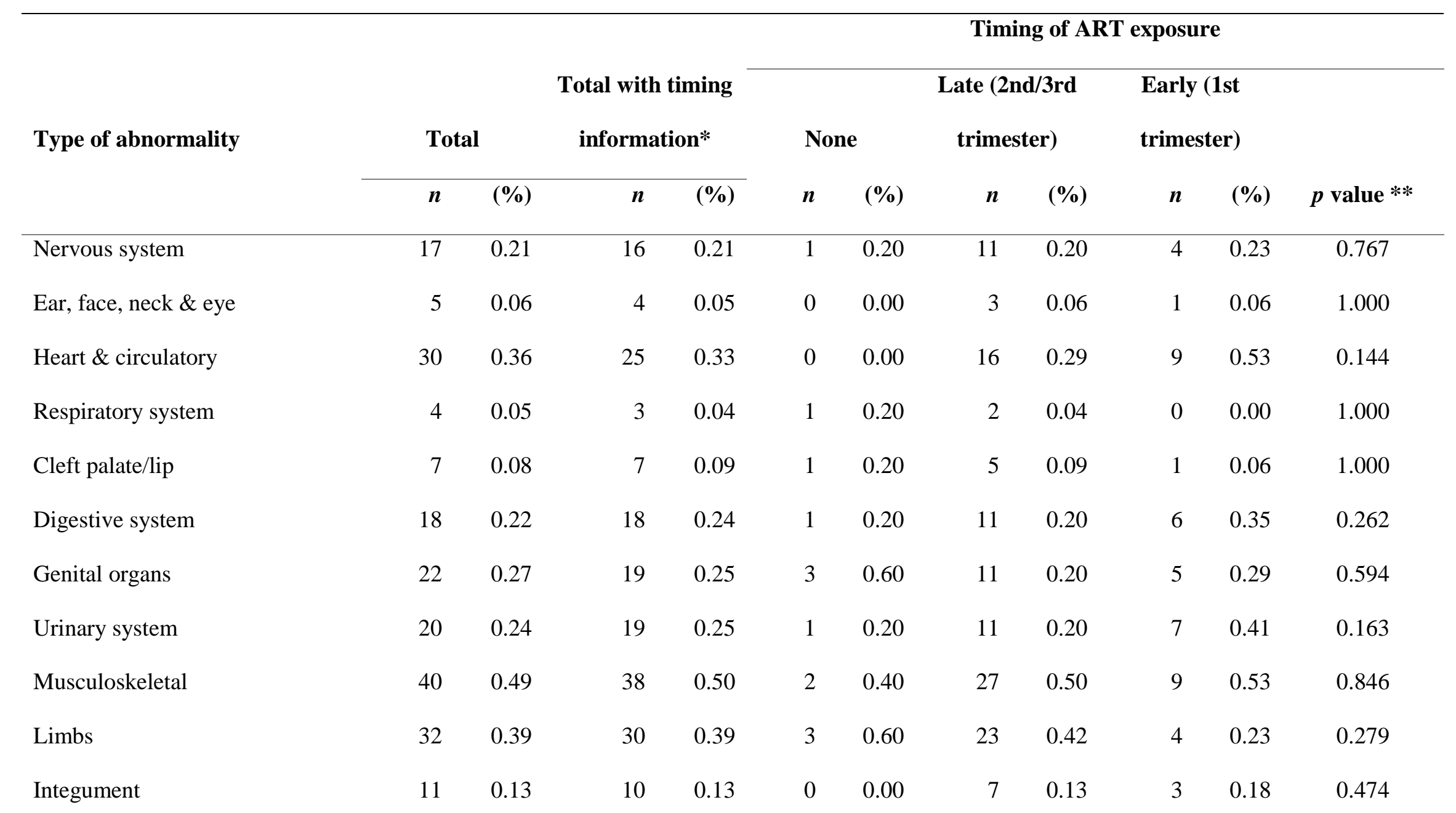




$\begin{array}{lrrrrrrrrrrr}\text { Chromosomal } & 21 & 0.25 & 20 & 0.26 & 1 & 0.20 & 16 & 0.29 & 3 & 0.18 & 0.594 \\ \text { Other \& unspecified anomalies } & 2 & 0.02 & 2 & 0.03 & 0 & 0.00 & 1 & 0.02 & 1 & 0.06 & 0.397 \\ \text { Type not specified } & 3 & 0.04 & 3 & 0.04 & 0 & 0.00 & 3 & 0.06 & 0 & 0.00 & 1.000 \\ \text { Total congenital abnormalities } & 232 & 2.81 & 214 & 2.80 & 14 & 2.81 & 147 & 2.71 & 53 & 3.10 & \\ \text { Total infants } & 8242 & & 7633 & & 498 & & 5427 & & 1708 & \end{array}$

* Excludes 18 infants with abnormalities for whom information on timing of treatment was not available

** Fisher's exact test for comparison of 1st trimester exposure with late or no exposure 


\section{Reference List}

(1) Townsend CL, Cortina-Borja M, Peckham CS, de RA, Lyall H, Tookey PA. Low rates of mother-to-child transmission of HIV following effective pregnancy interventions in the United Kingdom and Ireland, 2000-2006. AIDS 2008; 22(8):973-981.

(2) Townsend CL, Cortina-Borja M, Peckham CS, Tookey PA. Trends in management and outcome of pregnancies in HIV-infected women in the UK and Ireland, 1990-2006. BJOG 2008.

(3) European Collaborative Study. Does highly active antiretroviral therapy increase the risk of congenital abnormalities in HIV-infected women? J Acquir Immune Defic Syndr 2005; 40(1):116-118.

(4) Watts DH, Li D, Handelsman E, Tilson H, Paul M, Foca M et al. Assessment of birth defects according to maternal therapy among infants in the Women and Infants Transmission Study. J Acquir Immune Defic Syndr 2007; 44(3):299-305.

(5) Watts DH, Covington DL, Beckerman K, Garcia P, Scheuerle A, Dominguez K et al. Assessing the risk of birth defects associated with antiretroviral exposure during pregnancy. Am J Obstet Gynecol 2004; 191(3):985-992.

(6) Antiretroviral Pregnancy Registry Steering Committee. Antiretroviral Pregnancy Registry International Interim Report for 1 January 1989 through 31 July 2008. 2008. Available at: http://www.apregistry.com/. [Accessed 10 November 2008]. 
(7) De Santis M, Cavaliere AF, Caruso A, Villa P, Tamburrini E, Cauda R et al. Hemangiomas and other congenital malformations in infants exposed to antiretroviral therapy in utero. JAMA 2004; 291(3):305.

(8) Fundaro C, Genovese O, Rendeli C, Tamburrini E, Salvaggio E. Myelomeningocele in a child with intrauterine exposure to efavirenz. AIDS 2002; 16(2):299-300.

(9) Townsend CL, Tookey PA, Cortina-Borja M, Peckham CS. Antiretroviral therapy and congenital abnormalities in infants born to HIV-1-infected women in the United Kingdom and Ireland, 1990 to 2003. J Acquir Immune Defic Syndr 2006; 42(1):91-94.

(10) World Health Organization. International Statistical Classification of Diseases and Related Health Problems, 1989 Revision. Geneva: World Health Organization; 1992.

(11) EUROCAT. EUROCAT Special Report. The environmental causes of congenital anomalies: a review of the literature [online]. 2004. Available at: www.eurocat.ulster.ac.uk/pubdata. [Accessed 10 November 2008].

(12) EUROCAT. 1980-2006 Prevalence Data Tables. 2008. Available at: www.eurocat.ulster.ac.uk/pubdata/tables.html. [Accessed 10 November 2008]. 